147 47

\section{THE INDICATIONS AND OUTCOMES OF BALLOON AORTIC VALVULOPLASTY: A SINGLE CENTRE EXPERIENCE}

S Krishnamoorthy, S Rowley, L Barth, E Wade, J Nolan, M Gunning University Hospitals of North Staffordshire

doi:10.1136/heartjnl-2013-304019.147

Background Treatment of patients with severe aortic stenosis with transcatheter aortic valve implantation (TAVI) has led to the revival of balloon aortic valvuloplasty (BAV). We assessed the indications, immediate outcome and durability following BAV.

Methods A retrospective analysis of the practice at this institution between 2009 and 2012 was performed. Demographic, clinical, procedural and follow-up data were collected.

Results 63 patients with severe symptomatic aortic stenosis underwent BAV. All had appreciable co-morbidity. The median age was $80(75-86)$ years, $41(65.1 \%)$ were male. Of the entire cohort $22 \%$ were diabetic, $11 \%$ had a history of bypass surgery, $27 \%$ had previous stroke, $27 \%$ stage IV renal failure, $33 \%$ has severe airways disease and $52.4 \%$ were in atrial fibrillation. With regard to echocardiographic variables; the mean peak gradient pre-procedure was $66.1 \pm 19.3 \mathrm{~mm} \mathrm{Hg}$, mean aortic valve area was $0.61 \pm 0.2 \mathrm{~cm}^{2}$. Most had impaired left ventricular systolic function, mean ejection fraction $42.6 \pm 18 \%$. BAV was performed as a bridge to TAVI in 28 (44\%), surgical aortic valve replacement in $23(37 \%)$ and as a standalone therapy in 12 (19\%) patients.

Significant complications occurred in $4(6.4 \%)$ of patients; of whom 1 had peri-procedural MI, 1 had renal failure, 1 cardiac tamponade and 1 had a retroperitoneal bleed. No patient suffered significant aortic regurgitation, stroke, nor required PPM implantation. Although there was no immediate peri-procedural mortality, 4 (6.4\%) patients died of progressive deterioration later during the same admission. With respect to the procedural success, there was a significant reduction observed in the mean peak gradient to $50.2 \pm 18.1 \mathrm{~mm}$ of $\mathrm{Hg}(\mathrm{p}<0.001)$ compared to the baseline, as well as some improvement in the calculated valve area to 0.96 $\pm 0.3 \mathrm{~cm}^{2}(\mathrm{p}<0.001)$ following BAV. On survival analyses, $87.3 \%$ $77.8 \%, 74.6 \%$ and $73 \%$ were alive at 3-months, 6-months, 1-year and 2-years respectively, following BAV with or without destination therapy. Of the standalone therapy group, 8/12 (66.7\%) patients were successfully discharged home with crude survival rates of $100 \%, 100 \%, 87.5 \%$ and $62.5 \%$ at 1 -month, 3 -months, 6-months and 1-year respectively.

Conclusions BAV is a useful adjunct to allow definitive therapy for severe aortic stenosis in patients with significant co-morbidity. Even in those patients who are unsuitable for TAVI or surgical valve replacement, and who have the highest attendant risk for valve intervention, standalone $\mathrm{BAV}$ can provide an effective treatment. 